\title{
Mid-infrared upconversion spectroscopy
}

Tidemand-Lichtenberg, Peter; Dam, Jeppe Seidelin; Andersen, H. V.; Høgstedt, Lasse; Pedersen, Christian

Published in:

Journal of the Optical Society of America B: Optical Physics

Link to article, DOI:

10.1364/JOSAB.33.000D28

Publication date:

2016

Document Version

Publisher's PDF, also known as Version of record

Link back to DTU Orbit

Citation (APA):

Tidemand-Lichtenberg, P., Dam, J. S., Andersen, H. V., Høgstedt, L., \& Pedersen, C. (2016). Mid-infrared upconversion spectroscopy. Journal of the Optical Society of America B: Optical Physics, 33(11), D28-D35. https://doi.org/10.1364/JOSAB.33.000D28

\section{General rights}

Copyright and moral rights for the publications made accessible in the public portal are retained by the authors and/or other copyright owners and it is a condition of accessing publications that users recognise and abide by the legal requirements associated with these rights.

- Users may download and print one copy of any publication from the public portal for the purpose of private study or research.

- You may not further distribute the material or use it for any profit-making activity or commercial gain

- You may freely distribute the URL identifying the publication in the public portal

If you believe that this document breaches copyright please contact us providing details, and we will remove access to the work immediately and investigate your claim. 


\title{
Mid-infrared upconversion spectroscopy
}

\author{
P. Tidemand-Lichtenberg, ${ }^{1}$ J. S. Dam, ${ }^{2}$ H. V. Andersen, ${ }^{3}$ L. Høgstedt, ${ }^{2}$ and C. Pedersen ${ }^{1, *}$ \\ ${ }^{1}$ DTU Fotonik, Technical University of Denmark, DK-4000 Roskilde, Denmark \\ ${ }^{2}$ IRSee ApS, Frederiksborgvej 399, 4000 Roskilde, Denmark \\ ${ }^{3}$ Foss Analytical A/S, Foss Allé 1, DK-3400 Hillerød, Denmark \\ *Corresponding author: chrp@fotonik.dtu.dk
}

Received 25 July 2016; revised 15 September 2016; accepted 18 September 2016; posted 19 September 2016 (Doc. ID 272260); published 10 October 2016

\begin{abstract}
Mid-infrared (MIR) spectroscopy is emerging as an attractive alternative to near-infrared or visible spectroscopy. MIR spectroscopy offers a unique possibility to probe the fundamental absorption bands of a large number of gases as well as the vibrational spectra of complex molecules. In this paper we discuss non-collinear upconversion as a means for obtaining MIR spectra in the 5-10 $\mathrm{m}$ range with a resolution better than $20 \mathrm{~cm}^{-1}$ over the full interval using four discrete phase-match settings. A theoretical treatment of non-collinear upconversion is given and two different experimental implementations are tested. () 2016 Optical Society of America
\end{abstract}

OCIS codes: (300.6340) Spectroscopy, infrared; (300.6190) Spectrometers; (190.7220) Upconversion; (190.4410) Nonlinear optics, parametric processes.

http://dx.doi.org/10.1364/JOSAB.33.000D28

\section{INTRODUCTION AND BACKGROUND}

The mid-infrared (MIR) region extending from 2.5 to about $25 \mu \mathrm{m}$ is less explored than the visible $(400-700 \mathrm{~nm})$ and the near-infrared (NIR, 700-2500 nm) wavelength range. The corresponding MIR photon energy levels are too small to facilitate atomic electron transitions, but rather match vibrational or rotational energy transitions of gasses or complex molecules; hence the MIR region is linked to vibrational spectroscopy. In the 4 to $8 \mu \mathrm{m}$ range, fundamental absorption bands of many gas molecules can be probed, such as $\mathrm{CO}_{2}, \mathrm{NO}_{\mathrm{X}}$, and $\mathrm{SO}_{2}$ [1]. The 7 to $11 \mu \mathrm{m}$ region called the "fingerprint region" contains unique spectral features of complex compounds. Examples are diverse and include, for instance, pharmaceutical products [2], soil contaminants [3], polymer products [4], exoplanet search [5], or biomedical applications [6].

An obstacle for efficient exploitation of this attractive wavelength region has been a lack of good MIR light sources and low-noise MIR detectors. However, recent advancements within MIR light sources have changed this situation drastically with the advent of the supercontinuum light source [7], the quantum cascaded laser (QCL) [8], and MIR frequency combs [9] as replacements for, or complements to, traditional globars or MIR optical parametric oscillators (OPOs) [10]. In strong contrast hereto, low noise, fast and efficient MIR detector technology still lags behind. Traditional MIR detectors, such as the InSb or the mercury cadmium telluride (MCT) detector, both suffer from significant dark noise induced by thermal charge carriers in the detector material overlapping the detectors absorption band and from unwanted incident blackbody radiation. In both cases the noise originates from the finite temperature (e.g., room temperature) of matter. Consequently these detectors have to be cooled to cryogenic temperatures in order to operate with low noise [11].

This is very different compared to visible or near-infrared detectors. Here the detector energy levels are well above the photon energy of room temperature radiation; hence, the thermal induced noise is extremely small. Moreover, silicon detector technology is fast, highly efficient, and cheap.

Different types of instrumentation have been deployed for MIR spectroscopy. The most prominent and widely used is the infrared Fourier transform system (FTIR) [12-14]. A major obstacle for industrial applications, or use in harsh environment, is the moving mirror used to generate the FTIR spectrum. This mirror has to be moved smoothly over substantial lengths, and is thus susceptible to mechanical vibrations deteriorating the precision of the instrument. Alternatively, dispersive infrared spectrophotometers, which rely on diffraction gratings for dispersion of radiation, can be deployed [15]. More recent systems retrieve MIR spectral information from a combination of a broadband light source, e.g., MIR frequency comb, OPO or tunable QCLs, and upconversion detection [16-19]. In these implementations the spectrum is obtained from the spectral properties of the light source or by use of NIR spectroscopy of the upconverted light.

A first objective of this work is exploitation of MIR upconversion in the $5-10 \mu \mathrm{m}$ range for low noise detection of MIR signals. Upconversion is a sum-frequency generation process, in a highly imbalanced version where a strong pump laser interacts with a faint MIR signal transferring the MIR information to 
the near-infrared or visible wavelength range, and is hence detectable by highly efficient, sensitive Si-based photon counters or CCD cameras. Since the upconversion process is a second-order nonlinear effect no absorption in the crystal is involved. According to Kirchhoff's law [20] the emissivity of a transparent crystal is zero, hence it will not emit blackbody radiation leading to detrimental dark optical photon noise. Unwanted radiation from the crystals surroundings will also be strongly filtered in space and frequency due the phase-match requirement for efficient detection [21]. For these reasons upconversion has room temperature single-photon detection capability $[22,23]$.

A second objective of this work is to extract spectral information from the upconversion process over a broad span of wavelengths. The phase-match condition will be generalized to include non-collinear upconversion, and it will be shown that the corresponding upconverted images contain spectral information radially encoded in the images [23].

Moving toward longer wavelengths the selection of commercially available nonlinear crystals is limited. In this paper silver gallium sulfide $\mathrm{AgGaS}_{2}$ (AGS) is used, as it is available from several vendors and has reasonable parameter for upconversion in the 5-10 $\mu \mathrm{m}$ range. The useful transparency range of the material is from $850 \mathrm{~nm}$ to $11 \mu \mathrm{m}$. The material can phasematch over the full spectral range using $1064 \mathrm{~nm}$ as the mixing laser, giving an upconverted signal in the $870-960 \mathrm{~nm}$ range, appropriate for CCD camera detection. A single upconverted image, corresponding to a definite setting of the phasematch condition will contain spectral information over a large span of wavelengths $(\sim 1 \mu \mathrm{m})$ without any phase-match tuning. Combining several specific phase-match settings the 5 to $10 \mu \mathrm{m}$ range can be covered. Alternative materials may include lithium thioindate $\left(\mathrm{LiInS}_{2}\right)$, which can phase-match in the same range but with significantly lower nonlinear coefficient of $d_{\text {eff }} \approx 7 \mathrm{pm} / \mathrm{V}$, or silver gallium selenide $\left(\mathrm{AgGaSe}_{2}\right)$, which, however, would require a longer mixing wavelength in order to phase-match; hence, the upconverted signal would also be at longer wavelength beyond the detection range of Si-based detectors. This material, however, has a higher nonlinear coefficient of $d_{\text {eff }} \approx 30 \mathrm{pm} / \mathrm{V}$.

The paper is structured in the following way: first, the theory behind upconversion spectroscopy is described in Section 2, starting with the essential phase-matching properties of AGS, considering non-collinear interaction for different orientations of the nonlinear material. Then the obtainable spectral resolution is evaluated in Section 2.B, and the possibility to increase spectral coverage by multiplexing of several channels is investigated in Section 2.C.

In Section 3, the first experimental demonstration is described, using angle tuning of the nonlinear material in order to cover the full spectral range from 5 to $10 \mu \mathrm{m}$. The full spectral coverage is obtained by taking four measurements with different angular orientations of the nonlinear material and subsequently stitching the spectral data together.

Finally, the full spectral coverage is demonstrated in a single measurement, using a system with four spatially separated parallel channels in Section 4. The work is concluded in Section 5.

\section{THEORY OF NONLINEAR FREQUENCY UPCONVERSION}

Considering sum-frequency mixing for spectroscopy, the energy conservation condition strictly dictates the relation between the IR input and the upconverted signal. Assuming that the mixing field is single frequency, the frequency conversion process simply transfers the spectral content from IR input to the upconverted NIR spectral region. However, in order to obtain efficient frequency conversion, the phase-match condition (momentum conservation) also needs to be fulfilled. Denoting the input signal (IR), the mixing laser field (La) and the upconverted signal (NIR), the energy conservation and phase-match condition can be written as

$$
\begin{gathered}
\frac{1}{\lambda_{\mathrm{NIR}}}=\frac{1}{\lambda_{\mathrm{La}}}+\frac{1}{\lambda_{\mathrm{IR}}}, \\
\mathbf{k}_{\mathrm{NIR}}=\mathbf{k}_{\mathrm{La}}+\mathbf{k}_{\mathrm{IR}} .
\end{gathered}
$$

The wavelength is $\lambda_{i}$, where index $i=\mathrm{IR}, \mathrm{NIR}, \mathrm{La}$, denotes the three interacting fields, $\mathbf{k}_{i}$ denotes the corresponding wave vectors. In the following, different types of phase-matching will be evaluated for frequency upconversion using a laser field at around $1 \mu \mathrm{m}$ and $\mathrm{AgGaS}_{2}$ (AGS) as the nonlinear material, including non-collinear interaction. The Sellmeier equations of Ref. [24] will be used in the numerical examples. Figure 1 illustrates the coordinates and parameters used throughout the phase-match calculations. In the following calculations $u_{i}$ is the internal angle between the $\mathbf{k}_{i}$-vector projected to the $x z$-plane, and the direction of the mixing laser field. The corresponding angle in the $y z$-plane is called $v_{i}$. The mixing laser field inside the crystal is considered parallel to the $z$-axis., i.e., for collinear interaction $u_{\mathrm{IR}}=u_{\mathrm{NIR}}=0$ and $v_{\mathrm{IR}}=v_{\mathrm{NIR}}=0$.

Considering the nonlinear material, the cutting angle of the crystal, relative to the $\hat{c}$-axis is denoted $\theta_{\text {cut }}$, while the rotation angle of the nonlinear crystal is called $\rho_{c}$. Both the cutting angle and crystal rotation are assumed to be in the $x z$-plane. In order to calculate the phase-mismatch along the $z$-axis (parallel to the mixing laser), the angle between the $\mathbf{k}_{i}$-vectors of the interacting fields and the $z$-axis is found as the dot-product, $\cos \left(\rho_{i}\right)=$ $\hat{k}_{i} \cdot \hat{k}_{\text {La }}$, where index $i$ denotes the IR input or the upconverted NIR field and $\hat{k}_{i}$ indicates a unit vector in the direction of $\mathbf{k}_{i} . \rho_{i}$ describe internal angles in the crystal:

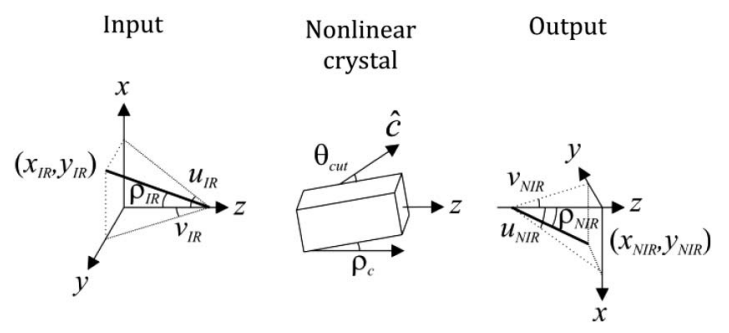

Fig. 1. Illustration of the coordinate system and parameters used in the phase-match calculations. $u_{i}$ is the internal angle between the $\mathbf{k}_{i}$-vector projected to the $x z$-plane, and the direction of the mixing laser field. The corresponding angle in the $y z$-plane is called $v_{i}$. The mixing laser field inside the crystal is considered parallel to the $z$-axis. 


$$
\cos \left(\rho_{i}\right)=\frac{1}{\sqrt{\tan ^{2}\left(u_{i}\right)+\tan ^{2}\left(v_{i}\right)+1}} .
$$

The phase-mismatch can now be evaluated along the $z$-axis, as well as in the transverse plane, $T$ :

$$
\begin{gathered}
\Delta k_{z}=2 \pi\left(\frac{n_{\mathrm{NIR}}}{\lambda_{\mathrm{NIR}}} \cdot \cos \left(\rho_{\mathrm{NIR}}\right)-\frac{n_{\mathrm{La}}}{\lambda_{\mathrm{La}}}-\frac{n_{\mathrm{IR}}}{\lambda_{\mathrm{IR}}} \cdot \cos \left(\rho_{\mathrm{IR}}\right)\right), \\
\Delta k_{T}=2 \pi\left(\frac{n_{\mathrm{NIR}}}{\lambda_{\mathrm{NIR}}} \cdot \sin \left(\rho_{\mathrm{NIR}}\right)-\frac{n_{\mathrm{IR}}}{\lambda_{\mathrm{IR}}} \cdot \sin \left(\rho_{\mathrm{IR}}\right)\right) .
\end{gathered}
$$

As a result of dispersion in the nonlinear crystal, described by the Sellmeier equations, it is not possible to obtain phasematching if all fields are polarized along the same axis. Typically birefringent phase-matching is divided into two groups: Type I, where the two input fields are parallel polarized and the generated field is orthogonal to the inputs, and Type II, where the two input beams are orthogonally polarized.

\section{A. Non-collinear Birefringent Phase-Matching}

In the following, Type II phase-match will be considered, i.e., where the input IR and the upconverted NIR fields are extraordinary polarized in the nonlinear material, and the laser field is ordinary polarized $\left(o_{\mathrm{IR}}+o_{\mathrm{La}} \rightarrow e_{\mathrm{NIR}}\right)$. AGS is a uniaxial material, hence the refractive index along the crystallographic axis can be simplified as $n_{o}=n_{a}=n_{b}$, where $n_{o}$ is the ordinary index of refraction, and $n_{e}=n_{c}$ is the extraordinary index of refraction; index $a, b$, and $c$ are the principal axis of the nonlinear material [25]. The extraordinary index of refraction depends on the angle of propagation relative to the $\hat{c}$-axis of the material according to [25]

$$
\frac{1}{n_{e}^{2}\left(\theta_{i}, \lambda_{i}\right)}=\frac{\sin ^{2}\left(\theta_{i}\right)}{n_{e}^{2}\left(\lambda_{i}\right)}+\frac{\cos ^{2}\left(\theta_{i}\right)}{n_{o}^{2}\left(\lambda_{i}\right)},
$$

where the subscript $i$ refers to the incident IR field or the upconverted NIR field.

Again the dot-product $\cos \left(\theta_{i}\right)=\hat{k}_{i} \cdot \hat{c}$, is used to calculate the angle between the respective $\mathbf{k}_{i}$-vectors and the $\hat{c}$-axis of the nonlinear material. The propagation angles can now be written as

$$
\cos \left(\theta_{i}\right)=\frac{\cos \left(\theta_{\text {cut }}+u_{i}+\rho_{c}^{*}\right)}{\sqrt{1+\cos ^{2}\left(u_{i}\right) \tan ^{2}\left(v_{i}\right)}},
$$

where $\rho_{c}^{*}=\arcsin \left(\sin \left(u_{\mathrm{La}}^{\mathrm{ex}}-\rho_{c}\right) / n\left(\lambda_{\mathrm{La}}\right)\right)$ describes how the rotation of the nonlinear material $\rho_{c}$ and an (externally) angled mixing laser field $u_{\mathrm{La}}^{\mathrm{ex}}$ impact the internal propagation angles. An example of the calculated extraordinary refractive index as a function of the internal propagation angles in the crystal is shown in Fig. 2.

In order to find the relation between phase-matched wavelengths and angles of propagation through the nonlinear material, both the transverse and longitudinal phase-mismatch in Eqs. (4) and (5) have to be zero. Considering the case where the IR and the NIR field are extraordinary polarized (Type II phase-match), conservation of the transverse momentum can be written in the following form:

$$
\sin \left(\rho_{\mathrm{IR}}\right) \frac{n_{e}\left(\theta_{\mathrm{IR}}, \lambda_{\mathrm{IR}}\right)}{\lambda_{\mathrm{IR}}}=\sin \left(\rho_{\mathrm{NIR}}\right) \frac{n_{e}\left(\theta_{\mathrm{NIR}}, \lambda_{\mathrm{NIR}}\right)}{\lambda_{\mathrm{NIR}}} .
$$

Furthermore, the input and output signals are in the same plane, i.e.,

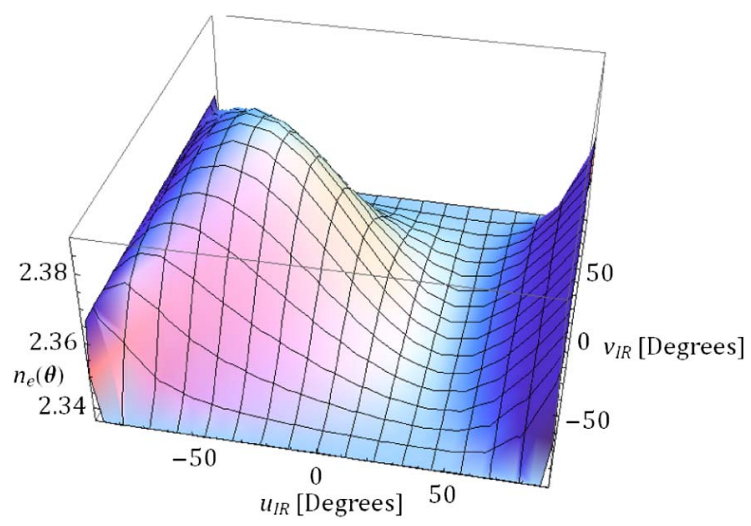

Fig. 2. Extraordinary refractive index as a function of propagation direction in the crystal, calculated at an IR wavelength of $6 \mu \mathrm{m}$ and a cut angle $\theta_{\text {cut }}=48^{\circ}$.

$$
\frac{\sin \left(v_{\mathrm{IR}}\right)}{\sin \left(u_{\mathrm{IR}}\right)}=\frac{\sin \left(v_{\mathrm{NIR}}\right)}{\sin \left(u_{\mathrm{NIR}}\right)}
$$

Solving equations for energy conservation [Eq. (1)], phasematch in the longitudinal [Eq. (4)], and transverse direction [Eq. (8)], and using that the input signal and the upconverted field is in the same plane [Eq. (9)], it is now possible to find corresponding phase-matched wavelengths and directions of propagation. Examples are shown in Fig. 3, where the phase-matched wavelengths have been calculated for mixing of the input IR signal with a $1064 \mathrm{~nm}$ laser. The phase-matched wavelengths are plotted for different rotations $\rho_{c}$, of the nonlinear material. Solid lines indicate angles in the $u$-direction and dashed lines are along the $v$-direction.

The general solution is almost concentric circles of constant wavelength, as can be seen in Fig. 4. Knowing the phase-match condition in the crystal, it is possible to calculate the corresponding wavelength and positions in the image plane that will be upconverted. Figure 4 shows matching image plane positions and wavelengths using 2-f imaging with a $50 \mathrm{~mm}$ focal length lens. The corresponding NIR output plane is calculated using 2-f imaging with a $100 \mathrm{~mm}$ focal length lens; see the experimental setup in Section 3 (Fig. 10).

An experimental demonstration of upconversion spectroscopy using the angle of the nonlinear material as the tuning parameter is described in Section 3.
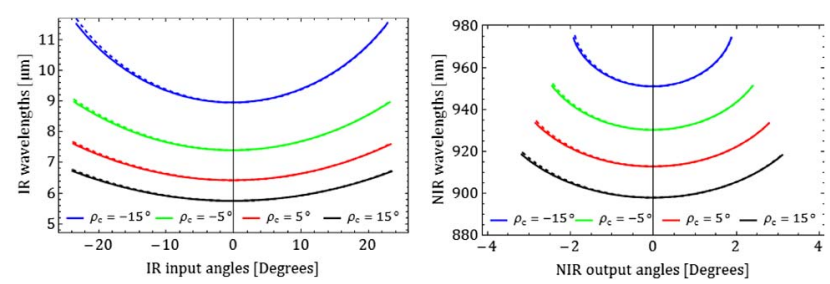

Fig. 3. Phase-matched wavelengths as a function of the direction of propagation of the interacting fields, plotted for different rotation angles of the nonlinear material. Solid lines shows external angles in the $u$-direction and dashed lines are the external angles in the $v$-direction. All calculations are performed for $\theta_{\text {cut }}=48^{\circ}$. 

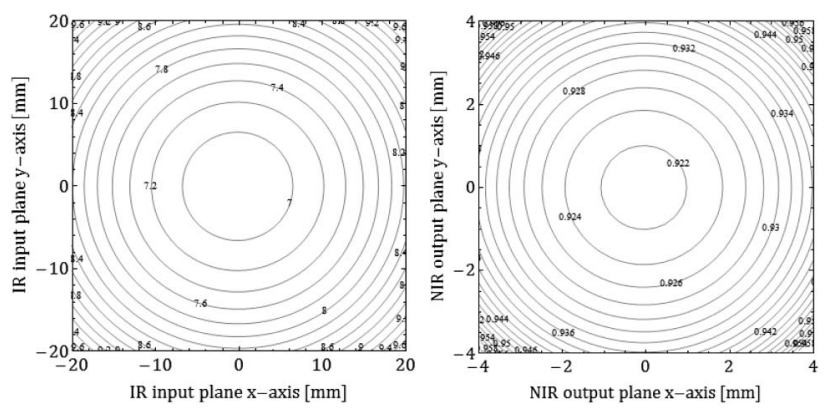

Fig. 4. Phase-matched wavelengths as a function of positions in the input plane using a $50 \mathrm{~mm}$ focal length lens and output plane using a $100 \mathrm{~mm}$ lens.

\section{B. Spectral Resolution}

In order to use the setup for spectral measurements with a broadband light source, the spectral resolution of the detection system is an essential parameter. The best spectral resolution that can be obtained using a broadband light source combined with upconversion detection is limited by the length of the nonlinear material, given a spectral resolution set by the acceptance bandwidth of the nonlinear conversion process.

Considering plane wave interaction, the spectral acceptance parameter can be derived directly from the energy and momentum conservation Eqs. (1) and (2). Taking the derivative of the phase-mismatch with respect to the IR wavelength, the value where the conversion efficiency has decreased to half of the peak value can be found. This can be evaluated to give the full width at half-maximum (FWHM) spectral acceptance bandwidth $\Delta \nu_{\text {IR }}$ for the IR signal $[25,26]$ :

$$
\begin{aligned}
\Delta \nu_{\mathrm{IR}}= & \frac{0.886}{L} \mid n_{e}\left(\theta_{\mathrm{IR}}, \lambda_{\mathrm{IR}}\right)-n_{e}\left(\theta_{\mathrm{NIR}}, \lambda_{\mathrm{NIR}}\right) \\
& -\lambda_{\mathrm{IR}} \frac{\partial n_{e}\left(\theta_{\mathrm{IR}}, \lambda_{\mathrm{IR}}\right)}{\partial \lambda_{\mathrm{IR}}}+\left.\lambda_{\mathrm{NIR}} \frac{\partial n_{e}\left(\theta_{\mathrm{NIR}}, \lambda_{\mathrm{NIR}}\right)}{\partial \lambda_{\mathrm{NIR}}}\right|^{-1},
\end{aligned}
$$

where $L$ is the length of the nonlinear crystal. Figure 5 shows the numerically calculated FWHM bandwidth of the upconverted IR wavelengths considered in Fig. 3.

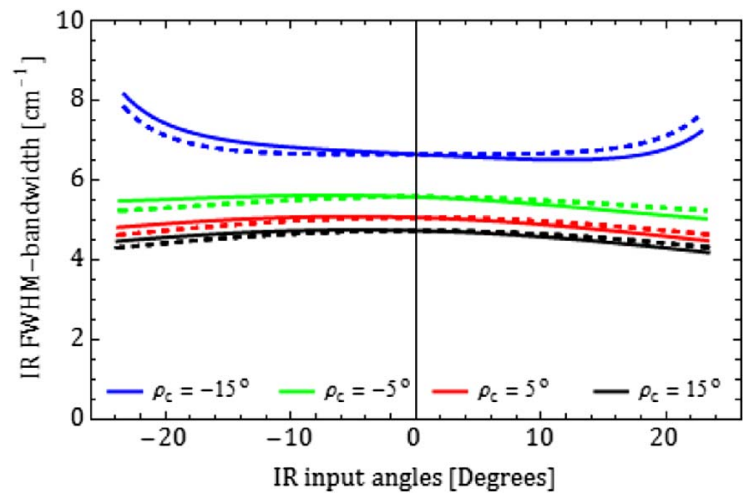

Fig. 5. Numerical calculation of the full width half-maximum acceptance bandwidth for the IR signal in a $10 \mathrm{~mm}$ long AGS crystal. The colored lines represent different crystal rotations (wavelength ranges); the solid and dashed lines represent the $u$ - and $v$-directions, respectively.
Evaluating larger non-collinear angles in the upconversion process, it was shown in [21] that the spectral resolution is limited by the non-collinear interaction and the finite width of the mixing laser beam, rather than the length and dispersion parameters of the nonlinear material. Hence, the spectral resolution deteriorates as angles increase [27].

In order to measure the full 5-10 $\mu \mathrm{m}$ range, a series of measurements has to be performed, using different orientations of the nonlinear material. The full spectrum is then found by stitching the individual spectra together. This results in longer acquisition time and the need for a rotation stage for control of the nonlinear material.

\section{Wavelength Multiplexing}

The spectral coverage in a single measurement can be increased using several spatially separated parallel channels, as described in the following.

Here a four-channel system is considered, where each channel is defined by a specific phase-match condition. The mixing fields pass through the nonlinear material at four predetermined angles, and two different mixing wavelengths. Channels 1 and 2 are configured with a $1020 \mathrm{~nm}$ mixing field illuminating the nonlinear crystal at external angles of $\theta_{\mathrm{ch} 1}=-13.5^{\circ}$ and $\theta_{\mathrm{ch} 2}=$ $-3.75^{\circ}$ relative to the $z$-axis. Channels 3 and 4 are configured with a $980 \mathrm{~nm}$ mixing field forming external angles of $\theta_{\mathrm{ch} 3}=$ $3.75^{\circ}$ and $\theta_{\text {ch } 4}=13.5^{\circ}$ relative to the $z$-axis. The AGS crystal is configured for Type I phase-matching $\left(o_{\mathrm{IR}}+o_{\mathrm{La}} \rightarrow e_{\mathrm{NIR}}\right)$ $\theta_{\text {cut }}=50.5^{\circ}, \phi=45^{\circ}$, and $\rho_{c}=2.5^{\circ}$; see Fig. 6 for details.

The phase-match condition for a single channel was evaluated in Section 2.A. This analysis is now expanded to include more angles $u_{\mathrm{La}}^{\mathrm{ex}}$ of propagation for the input mixing laser field, resulting in phase-matched IR wavelengths as a function of input and output wavelengths, as shown in Fig. 7.

As can be seen from Fig. 7, the input IR signal for the different spectral components partly overlap in angle, whereas the upconverted wavelengths do not overlap, making it simple

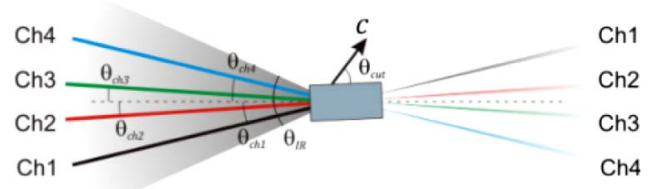

Fig. 6. Configuration of the four-channel system for full spectral coverage in a single measurement from 5 to $10 \mu \mathrm{m}$. The gray-shaded region indicates the illumination angles of the IR.
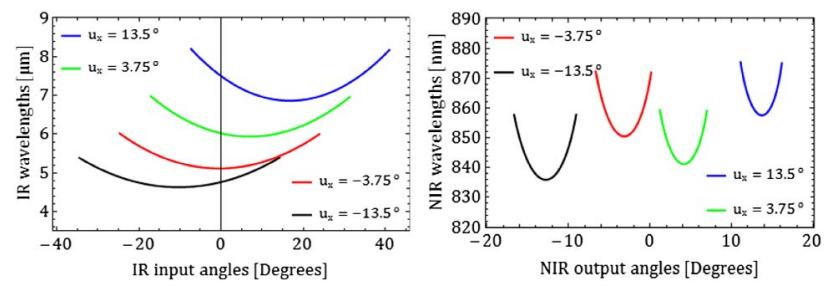

Fig. 7. Phase-matched IR wavelengths versus external input angles and the corresponding external angles of the upconverted signal. 


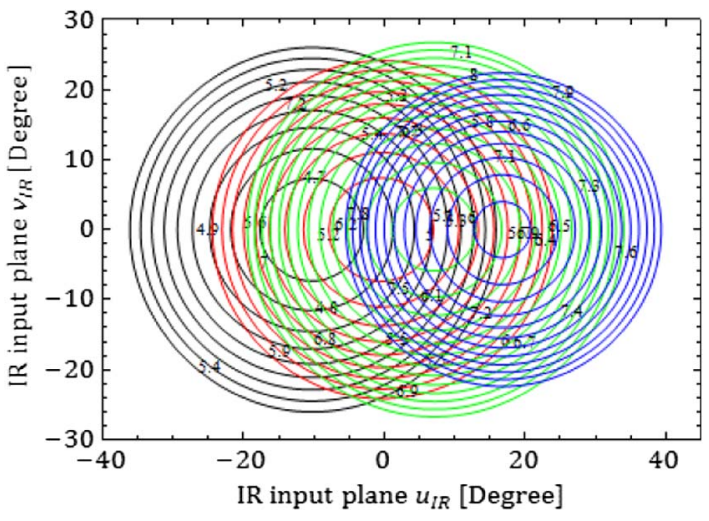

Fig. 8. Contour plots of the phase-matched IR wavelengths as a function of input angles.

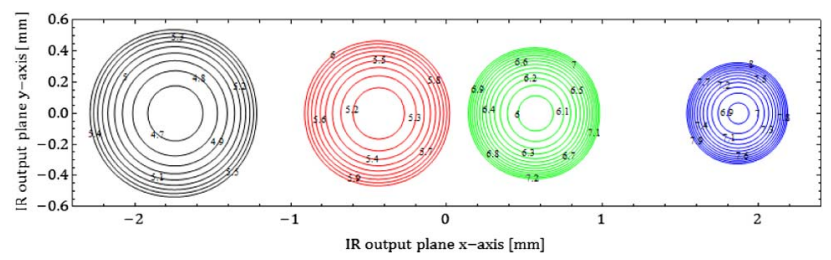

Fig. 9. Spatial distribution of the upconverted IR wavelengths at the detector plane using an $8 \mathrm{~mm}$ focal length lens.

to de-convolve the spectral components. This is further illustrated in Fig. 8, which shows the superposition of the phase-matched IR ring pattern for the four channels. The corresponding spatial distribution of the upconverted NIR side is shown in Fig. 9, where the contour lines indicate the phasematched IR wavelengths that have been upconverted.

An experimental demonstration of the upconversion-based spectrometer is described in Section 4.

Before moving to the experimental part, it is worth noting that in sum-frequency mixing there is a linear relation between the power of the input IR signal and the upconverted output signal, in strong contrast to second-harmonic generation. Furthermore, it is important that the upconverted power scales linearly with the mixing laser power, whereas it is independent of the focusing of the mixing laser, as long as the input IR signal is spatially incoherent [28].

\section{SPECTRAL MEASUREMENTS IN THE 5-10 $\mu$ M RANGE}

In this section a first experimental demonstration of upconversion spectroscopy in the $5-10 \mu \mathrm{m}$ range is described. A schematic layout of the setup is shown in Fig. 10.

The system consists of a $1070 \mathrm{~nm}, 10 \mathrm{~W}$ vertically polarized fiber laser, which is collimated to a beam size (radius) of approximatley $2 \mathrm{~mm}$. The mixing laser beam is passed through the nonlinear material (AGS), which is cut for Type II phasematching $\left(e_{\mathrm{IR}}+o_{\mathrm{La}} \rightarrow e_{\mathrm{NIR}}\right)$, as described in Section 2.A. The crystal is cut with an angle, $\theta_{\text {cut }}=48^{\circ}$, relative to the optical axis and $\phi=0^{\circ}$, giving an effective nonlinear coefficient, $d_{\text {eff }} \approx 16 \mathrm{pm} / \mathrm{V}$. The IR signal is generated by a

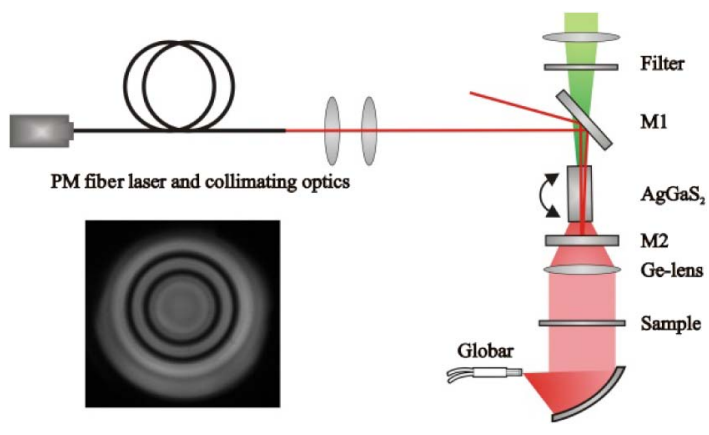

Fig. 10. Schematic layout of the 5 to $10 \mu \mathrm{m}$ upconversion setup. The nonlinear crystal is mounted on a rotation stage to allow for spectral tuning through angle rotation. The insert shows a typical image of the ratio between an image with a PS film sample and an image without a sample.

$4.5 \mathrm{~mm}$ diameter globar operating at $1100 \mathrm{~K}$, collimated by an off-axis parabolic mirror $(f=30 \mathrm{~mm})$, focused to the sample using a $40 \mathrm{~mm} \mathrm{CaF}$ lens, and re-collimated by a $100 \mathrm{~mm}$ $\mathrm{CaF}_{2}$ lens (not shown in Fig. 10). A Ge lens with a focal length of $25 \mathrm{~mm}$ serves a dual purpose, namely to act as a long pass (LP) filter to remove wavelengths below $2 \mu \mathrm{m}$ and to focus the IR light into the nonlinear material. Mirror M2 [high-reflection (HR) at 1064 , antireflection (AR) at $5-10 \mu \mathrm{m}$ on a $\mathrm{CaF}_{2}$ substrate] combines the IR signal and the mixing laser field. This layout allows for large angles of incidence of the IR signal to enter the nonlinear crystal, promoting a large spectral coverage in a single measurement; however, only the co-propagating fields contribute to the upconverted signal. The upconverted signal is separated from the mixing laser by mirror M1 (HR at $1064 / 45 \mathrm{deg}, \mathrm{AR}$ at $820-970 \mathrm{~nm} / 45 \mathrm{deg}$ ), filtered by LP850 and SP1000 nm filters and imaged onto the CCD camera (Thorlabs DCU224) with a $30 \mathrm{~mm}$ achromatic lens. An example of an upconverted image is shown in the insert of Fig. 10. The IR signal is passed through the test sample, in this case a polystyrene (PS) film; hence, the dark rings in the image indicate highly absorbing spectral features in the PS film.

The procedure for measurement of the absorbance of a sample, e.g., the PS film, is as follows: first, a background measurement is performed without any IR input signal in order to subtract background noise from the system, originating from dark noise in the camera or noise generated in the upconversion unit. Then the IR light source, i.e., the globar, is turned on and a reference measurement is obtained without the sample in the IR illumination path. This will eliminate the spectral imprint of the IR light source on the upconverted image caused by any spectral non-uniformity. Finally the sample is inserted and a measurement of the transmitted signal is performed. The background measurement is subtracted from each of the reference and signal measurements, and the ratio of these is calculated. A radial analysis of the ratioed images is performed using the phase-match theory outlined in Section 2.A, hence an absorbance spectrum of the sample can be calculated. Note that due to the circular symmetry the upconverted image contains redundant information, which can be used to increase the signal-to-noise ratio (SNR). The image acquisition and the 
subsequent analysis of the images are automated using a LabView program.

Combining four different crystal orientations $\rho_{c}$, it is possible to measure the full absorbance spectrum from 5 to $10 \mu \mathrm{m}$, thus covering a major part of the fingerprint region.

In order to obtain the full spectrum shown in Fig. 11, external IR angles of incidence up to $30^{\circ}$ are used. The cut angle of the nonlinear crystal was $\theta_{\text {cut }}=48^{\circ}$, and the specifications of the four individual channels are listed in Table 1.

One example of a $5-10 \mu \mathrm{m}$ spectral measurement is shown in Fig. 11.

As mentioned, the system is based on Type II phasematched, sum-frequency mixing, i.e., only the part of the IR field that is orthogonally polarized to the mixing laser is upconverted. The residual (unconverted part) of the IR signal is removed by filters allowing only the upconverted signal to reach the camera.

Using a $10 \mathrm{~W}$ mixing laser in the above measurement, each single measurement used an integration time of $1 \mathrm{~s}$ on the camera. The SNR over the $5-10 \mu \mathrm{m}$ interval, in an air sample, varied significantly. In this system we obtained an SNR of approximately 2000 at $1450 \mathrm{~cm}^{-1}$ (in the center of the image), decreasing to about 300 approaching the edge of the image, however giving a typical SNR of $>700$ over most of spectral region referring to a $1.2 \mathrm{~cm}^{-1}$ spectral bin resolution. Comparing to a typical FTIR system with a spectral resolution of $8 \mathrm{~cm}^{-1}$ this will have a SNR of 10.000 to 20.000 on air with $1 \mathrm{~s}$ integration time (see Fig. 12). For reference, the SNR measurement was also performed using a PS film as the sample; see the green graphs in Fig. 11.

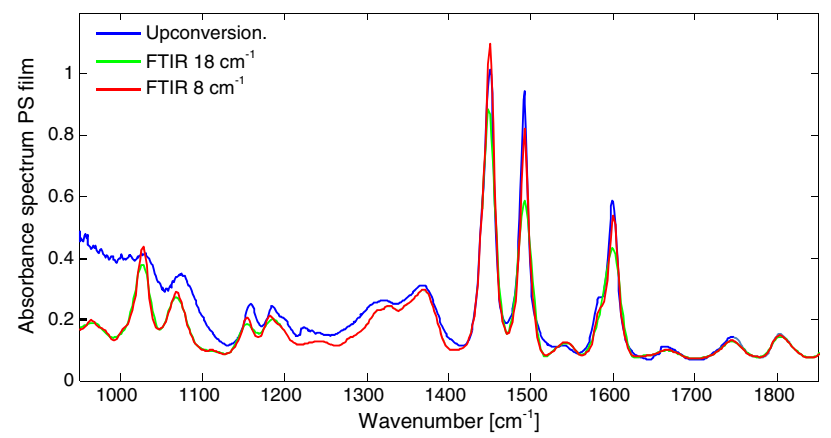

Fig. 11. Measured absorbance spectrum of a $0.038 \mathrm{~mm}$ thick PS film, combining measurements from four different crystal angles, $\rho_{c}$. The measured absorbance spectrum is compared to FTIR measurement with $8 \mathrm{~cm}^{-1}$ and $18 \mathrm{~cm}^{-1}$ spectral resolution, respectively.

Table 1. Specification of the Four Phase-Match Settings, i.e., Channels, Used to Cover the 5-10 $\mu \mathrm{m}$ Wavelength Range ${ }^{a}$

\begin{tabular}{lc}
$\begin{array}{l}\text { AGS Crystal } \\
\text { Angle }\left(\boldsymbol{\rho}_{\boldsymbol{c}}\right)\end{array}$ & $\begin{array}{c}\text { Wavelengths Phase-Matched } \\
\text { for Upconversion }\left(\lambda_{\text {IR }}\right)\end{array}$ \\
\hline$-11.3^{\circ}$ & $8.2 \mu \mathrm{m}-11.4 \mu \mathrm{m}$ \\
$1.9^{\circ}$ & $6.6 \mu \mathrm{m}-8.5 \mu \mathrm{m}$ \\
$10.7^{\circ}$ & $6.0 \mu \mathrm{m}-7.5 \mu \mathrm{m}$ \\
$18.9^{\circ}$ & $5.5 \mu \mathrm{m}-6.8 \mu \mathrm{m}$ \\
\hline
\end{tabular}

${ }^{a}$ The angle, $\rho_{c}$, is measured relatively to the $z$-axis.
The demonstrated system needs four different channels (crystal settings) in order to cover the full spectrum from 5 to $10 \mu \mathrm{m}$, using a mechnical rotation stage. In the following section a different approach is demonstrated giving the full spectral coverage in a single measurement, hence eliminating the need for mechanical tuning of the system.

\section{MULTI-CHANNEL WAVELENGTH SYSTEM}

In the following, a system comprising four parallel channels is considered. The channels are formed by combining two individual mixing laser fields, propagating through the nonlinear material at four different angles (see Section 2.C, Fig. 6). The mixing field in Channels 1 and 2 shares the same $600 \mathrm{~mW}$, $1020 \mathrm{~nm}$ polarization maintaining (PM) fiber-coupled diode laser (InnoLume/LD-1020-PM-600) using a 50/50 fiber beam splitter (AFW Technology). A similar configuration is used for Channels 3 and 4, using a $900 \mathrm{~mW}, 980 \mathrm{~nm}$ PM fiber-coupled diode laser (Thorlabs/BL976-PAG-900). Channels 1 and 4 are collimated by a fiber collimator from Thorlabs (F230APC-980) forming a focus with a diameter of $1 \mathrm{~mm}$ in the AGS crystal. Channels 2 and 3 are collimated to a slightly larger beam waist (F240APC-980), forming a focus diameter of $1.7 \mathrm{~mm}$ in the nonlinear material. The reason for choosing a smaller beam diameter for the channels with larger incident angles is determined from optical simulations of the lens forming the image on the camera, optimizing for best possible image formation on the camera. The mechanical layout is shown in Fig. 13.

The IR signal is focused by a $25 \mathrm{~mm}$ Ge lens (not shown in the figure) and combined with the four laser beams by the dichroic coupling mirror. The upconversion crystal is a $5 \times 5 \times 10 \mathrm{~mm}^{3}$ AGS crystal cut for Type I phase-matching $\left(o_{\mathrm{IR}}+o_{\mathrm{La}} \rightarrow e_{\mathrm{NIR}}\right)$ using $\theta_{\text {cut }}=50.5^{\circ}$ relative to the optical axis and $\phi=45^{\circ}$. The upconverted signals are imaged onto the camera (QHY5H-II from QHYCCD) using an $8 \mathrm{~mm}$ aspherical lens (\#68-139 from Edmund Optics) in a 2-f configuration, giving images as shown in Fig. 14.

In order to make a realistic measurement, the system was tested for gas sensing. A mixture of $100 \mathrm{ppm} \mathrm{SO}_{2}$ and $800 \mathrm{ppm} \mathrm{H}_{2} \mathrm{O}$ at $180^{\circ} \mathrm{C}$ was used as a test sample. The corresponding theoretically predicted transmission spectrum

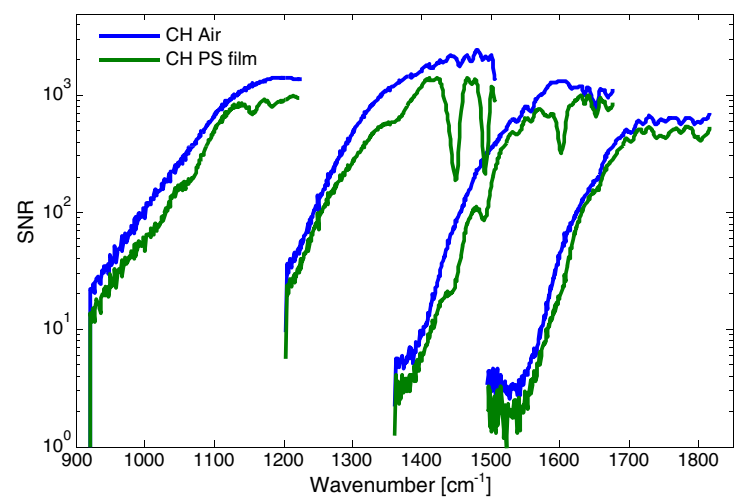

Fig. 12. SNR measured on air (blue) and PS film (green), respectively. The best SNR is obtained in the center of the images and reaches approximately 2000 at $1450 \mathrm{~cm}^{-1}$. The PS film is included for comparison. 


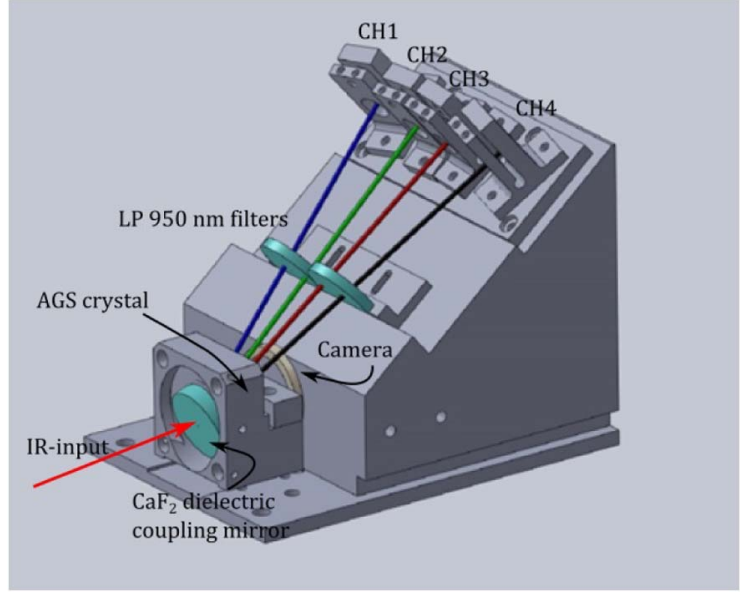

Fig. 13. Layout of four-beam multi-channel system. The colored rays indicate the mixing fields of the four channels.

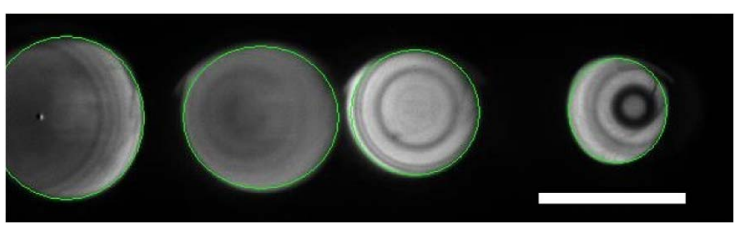

Fig. 14. Spatial distribution of the phase-matched IR wavelengths at the output plane using an $8 \mathrm{~mm}$ focal length lens. White scale bar is $1 \mathrm{~mm}$.

calculated by the HITRAN2012 database using SpectralCalc. com is shown in Fig. 15.

A globar was used as the IR light source, passing through a $50 \mathrm{~cm}$ long tube containing the hot gas mixture. As in Section 3, a radial image analysis of the signal and reference image provides the absorbance spectrum, as shown in Fig. 16.

This first demonstration shows a good correlation between the measured and theoretically predicted spectra, comparing Figs. 15 and 16, thus demonstrating the potential for gas spectroscopy. Using appropriate software for the chemometrics, it is

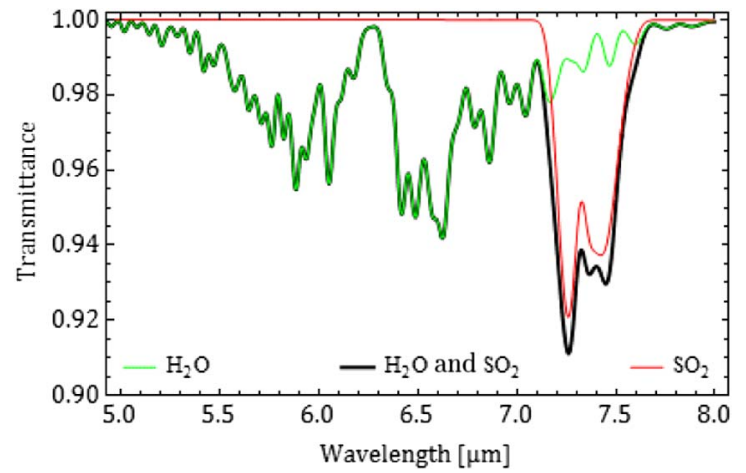

Fig. 15. Theoretical transmission spectrum of the hot gasses with an interaction length of $100 \mathrm{~cm}$ at $180^{\circ} \mathrm{C}$. Green graph, $800 \mathrm{ppm}$ $\mathrm{H}_{2} \mathrm{O}$; red graph, $100 \mathrm{ppm} \mathrm{SO}$; and black graph the mixed gasses, calculated from SpectalCalc.com (Hitran 2012 database), and a Gaussian instrument function, with a width of $40 \mathrm{~nm}$.

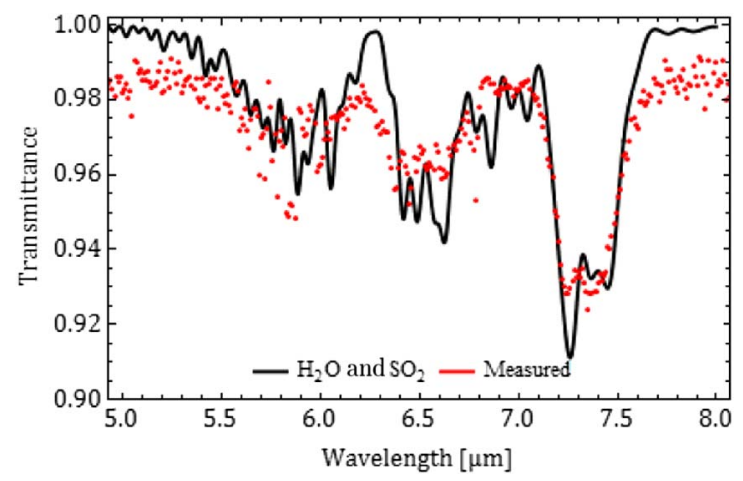

Fig. 16. Measured transmission spectrum compared to the theoretical spectrum from Fig. 15.

possible to derive the components present in a gas sample from this type of measurement. In this system we obtain a typical $\mathrm{S} / \mathrm{N}$ of about 1:500 in $15 \mathrm{~s}$. As a further example, measurement on a $\mathrm{SO}_{2}$ gas showed a precision of $<2 \mathrm{ppm}$. The spectral resolution is similar to that described in Section 3. Compared to the system described in Section 3, the multi-channel system represents a compact, robust, and integrated system with no moving parts, however, with significant lower mixing power, hence giving a correspondingly reduced SNR.

\section{CONCLUSION}

In this paper we have presented the theoretical apparatus for calculating the phase-match curves for non-collinear upconversion and shown that spectral information in a broad wavelength range (i.e., $1 \mu \mathrm{m}$ ) can be encoded radially in a single upconverted image. The spectral resolution is retrieved by the acceptance bandwidth dictated by the crystals phase-match condition. The resolution in our experiments is of the order of $10 \mathrm{~cm}^{-1}$ to $18 \mathrm{~cm}^{-1}$ depending on the specific MIR center wavelength. In order to spectrally cover the $5-10 \mu \mathrm{m}$ range, corresponding to the MIR spectral fingerprint region, four different channels were needed using silver gallium sulfide. A first implementation deployed four preselected angular crystal settings, each covering a part of the $5-10 \mu \mathrm{m}$ range. Actual absorbance measurement on a PS film showed a good similarity with corresponding FTIR measurements when using a spectral resolution of $8 \mathrm{~cm}^{-1}-18 \mathrm{~cm}^{-1}$ of the FTIR instrument. An experimental study on air provided detailed information of the SNR over the full wavelength range. Depending on the specific wavelength, the SNR can reach up to 2000 for a $1 \mathrm{~s}$ measurement and was above 700 over most of the 5-10 $\mu \mathrm{m}$ range. A second implementation was realized in order to remove the need for moving mechanical components. Such a system was accomplished using four channels, by replacing angle tuning of the AGS crystal with two different laser diodes, each divided into two beams propagating at different, but fixed angles. The four upconverted images were well separated on the CCD camera and could be analyzed individually. In order to test this configuration experimentally a mixture of $100 \mathrm{ppm} \mathrm{SO}_{2}$ and $800 \mathrm{ppm} \mathrm{H}_{2} \mathrm{O}$ at $180^{\circ} \mathrm{C}$ contained in a $50 \mathrm{~cm}$ long tube was illuminated by a globar in transmission. The measured spectra extending from 4.6 to $8 \mu \mathrm{m}$ were compared with a 
corresponding theoretical transmission spectrum displaying the expected spectral features. In conclusion, upconversion spectroscopy in the $5-10 \mu \mathrm{m}$ wavelength range is realized using no movable components and with a medium resolution and SNR showing a good potential for MIR spectroscopy.

Funding. Danish Maritime Fund (Røggassensor) (2014094-2); European Union's Horizon 2020 Research and Innovation Programme (Mid-TECH Project) (642661).

Acknowledgment. We want to acknowledge Foss Analytical A/S for valuable support of this study.

\section{REFERENCES}

1. N. Yamazoe and N. M. Environmental, "Environmental gas sensing," Sens. Actuators B 20, 95-102 (1994).

2. S. Wartewig and R. H. H. Neubert, "Application of mid-IR spectroscopy for the characterization of pharmaceutical," Adv. Drug Delivery Rev. 57, 1144-1170 (2005).

3. B. Ludwig, R. Nitschke, T. Terhoeven-Urselmans, K. Michel, and H. Flessa, "Use of mid-infrared spectroscopy in the diffuse-reflectance mode for the prediction of the composition of organic matter in soil and litter," J. Plant Nutr. Soil Sci. 171, 384-391 (2008).

4. D. Cai, A. Neyer, R. Kuckuk, and H. M. Heise, "Raman, mid-infrared, near-infrared and ultraviolet-visible spectroscopy of PDMS silicone rubber for characterization of polymer optical waveguide materials," J. Mol. Struct. 976, 274-281 (2010).

5. A. Morlok, C. M. Lisse, A. B. Mason, E. S. Bullock, and M. M. Grady, "Mid-infrared spectroscopy of components in chondrites: search for processed materials in young solar systems and comets," Icarus 231, 338-355 (2014).

6. A. Lux, R. Müller, M. Tulk, C. Olivieri, R. Zarrabeita, T. Salonikios, and B. Wirnitzer, "HHT diagnosis by mid-infrared spectroscopy and artificial neural network analysis," Orphanet J. Rare Dis. 8, 94 (2013).

7. R. Petersen, U. Møller, I. Kubat, B. Zhou, S. Dupont, J. Ramsay, T. Benson, S. Sujecki, N. Abdel-Moneim, Z. Tang, D. Furniss, A. Seddon, and O. Bang, "Mid-infrared supercontinuum covering the 1.4-13.3 $\mu \mathrm{m}$ molecular fingerprint region using ultra-high NA chalcogenide step-index fibre," Nat. Photonics 8, 830-834 (2014).

8. Y. Yao, A. J. Hoffman, and C. F. Gmachl, "Mid-infrared quantum cascade lasers," Nat. Photonics 6, 432-439 (2012).

9. A. Hugi, G. Villares, S. Blaser, H. C. Liu, and J. Faist, "Mid-infrared frequency comb based on a quantum cascade laser," Nature 492 , 229-233 (2012).

10. D. Creeden, P. A. Ketteridge, P. A. Budni, S. D. Setzler, Y. E. Young, J. C. McCarthy, K. Zawilski, P. G. Schunemann, T. M. Pollak, E. P. Chicklis, and M. Jiang, "Mid-infrared $\mathrm{ZnGeP}_{2}$ parametric oscillator directly pumped by a pulsed $2 \mu \mathrm{m}$ Tm-doped fiber laser," Opt. Lett. 33, 315-317 (2008).

11. A. Rogalski, Infrared Detectors, 2nd ed. (CRC Press, 2011).
12. M. J. Walsh, R. K. Reddy, and R. Bhargava, "Label-free biomedical imaging with mid-IR spectroscopy," IEEE J. Sel. Top. Quantum Electron. 18, 1502-1513 (2012).

13. F. Adler, P. Masowski, A. Foltynowicz, K. C. Cossel, T. C. Briles, I. Hartl, and J. Ye, "Mid-infrared Fourier transform spectroscopy with a broadband frequency comb," Opt. Express 18, 21861-21872 (2010).

14. E. D. Becker and T. C. Farrar, "Fourier transform spectroscopy," Science 178, 361-368 (1972).

15. D. L. Pavia, G. M. Lampman, G. S. Kriz, and J. A. Vyvyan, Introduction to Spectroscopy, 5th ed. (Cengage Learning, 2014).

16. J. Zhu, T. Mathes, A. D. Stahl, J. T. M. Kennis, and M. L. Groot, "Ultrafast mid-infrared spectroscopy by chirped pulse upconversion in $1800-1000 \mathrm{~cm}^{-1}$ region," Opt. Express 20, 10562-10571 (2012).

17. T. W. Neely, L. Nugent-Glandorf, F. Adler, and S. A. Diddams, "Broadband mid-infrared frequency upconversion and spectroscopy with an aperiodically poled $\mathrm{LiNbO}_{3}$ waveguide," Opt. Lett. 37, 4332-4334 (2012).

18. T. A. Johnson and S. A. Diddams, "MIR-IR frequency comb upconversion spectroscopy," in Conference on Lasers and Electro-Optics (CLEO) (Optical Society of America, 2010).

19. M. G. Hansen, I. Ernsting, S. V. Vasilyev, A. Grisard, E. Lallier, B. Gérard, and S. Schiller, "Robust, frequency-stable and accurate mid-IR laser spectrometer based on frequency comb metrology of quantum cascade lasers upconverted in orientation-patterned GaAs," Opt. Express 21, 27043-27056 (2013).

20. R. M. Goody and Y. L. Yung, Atmospheric Radiation: Theoretical Basis, 2nd ed. (Oxford University, 1989).

21. C. Pedersen, Q. Hu, L. Høgstedt, P. Tidemand-Lichtenberg, and J. S. Dam, "Non-collinear upconversion of infrared light," Opt. Express 22 , 28027-28036 (2014).

22. G. Temporão, S. Tanzilli, H. Zbinden, and N. Gisin, "Mid-infrared single-photon counting," Opt. Lett. 31, 1094-1096 (2006).

23. J. S. Dam, C. Pedersen, and P. Tidemand-Lichtenberg, "Room temperature mid-IR single photon spectral imaging," Nat. Photonics 6 , 788-793 (2012).

24. L. Wang, Z. Cao, H. Wang, H. Zhao, W. Gao, Y. Yuan, W. Chen, W. Zhang, Y. Wang, and X. Gao, "A widely tunable $(5-12.5 \mu \mathrm{m})$ continuous-wave mid-infrared laser spectrometer based on difference frequency generation in $\mathrm{AgGaS}_{2}$," Opt. Commun. 284, 358-362 (2011).

25. V. G. Dmitriev, G. G. Gurzadyan, and D. N. Nikogosyan, Handbook of Nonlinear Optical Crystals, 3rd ed. (Springer, 1999).

26. M. Fejer, G. Magel, D. Jundt, and R. Byer, "Quasi-phase-matched second harmonic generation: tuning and tolerances," IEEE J. Quantum Electron. 28, 2631-2654 (1992).

27. L. M. Kehlet, P. Tidemand-Lichtenberg, J. S. Dam, and C. Pedersen, "Infrared upconversion hyperspectral imaging," Opt. Lett. 40, 938-941 (2015).

28. J. S. Dam, C. Pedersen, and P. Tidemand-Lichtenberg, "Highresolution two-dimensional image upconversion of incoherent light," Opt. Lett. 35, 3796-3798 (2010). 